\title{
Coexistence of attractors in a laser diode with optical feedback from a large external cavity
}

\author{
C. Masoller \\ Instituto de Física, Facultad de Ciencias, T. Narvaja 1674, Montevideo, Uruguay \\ and Instituto de Física, Facultad de Ingenieria, Herrera y Reissig 565, Montevideo, Uruguay \\ (Received 31 January 1994; revised manuscript received 28 March 1994)
}

\begin{abstract}
The coexistence of several attractors in the coherent collapsed state of a laser diode with optical feedback is investigated. These attractors are unstable tori that bifurcate from different external cavity modes. As the feedback rate is increased, these tori undergo different types of quasiperiodic routes, such as frequency locking, period doubling, or the appearance of a third incommensurate frequency. In the fully developed coherent collapsed state, the tori are all unstable, and the phenomenon of intermittence appears. Lyapunov exponent calculations demonstrate that this state presents hyperchaotic and highdimensional dynamics. These results are explained qualitatively in terms of the multiattractor behavior found.
\end{abstract}

PACS number(s): 42.50.Lc, 05.45. $+\mathrm{b}$, 42.55.Px

\section{INTRODUCTION}

Optical feedback from an external cavity can dramatically modify the behavior of a semiconductor laser. While weak feedback levels result in linewidth narrowing and improved frequency stability, moderate to strong feedback levels might induce the laser to switch to a state of significant spectral broadening and dynamical complexity that has been called a coherent collapsed state.

Since a semiconductor laser can be expected to have some amount of feedback in almost all applications, e.g., reflections from fiber facets in optical fibers or reflections from the disk in a compact disk player, a lot of attention has been given to the study of the feedback phenomena induced by reflectors [1-13]. In addition to its practical importance, the rich variety of nonlinear phenomena observed, and the fact that the external cavity renders the system infinite dimensional, have made the external cavity laser a good candidate for the study of deterministic chaos in high-dimensional systems.

Several different regimes have been found for different values of the effective reflectivity and of the external cavity length.

We have recently studied [14] the effect of increasing the amount of feedback in the dynamics of a semiconductor laser with moderate feedback from a short external cavity, and found that the laser undergoes a quasiperiodic route interrupted by period doubling of a two-torus. In this contribution we present an in-depth investigation of the onset of a coherent collapsed state in a laser diode with weak optical feedback from an external cavity of several meters. We report the coexistence of several attractors, and we find that, when the laser operates in the coherent collapsed state, a switching between these unstable attractors occurs. We show that these unstable attractors are tori that were originated after Hopf bifurcations of different external cavity modes (ECM) of the laser. As a consequence, they have the same incommensurate frequencies and similar Fourier spectrum. However, we find that they have very different topological and stability properties and, therefore, they might be easily distinguished by calculating their Poincaré section $[15,16]$ and Lyapunov spectrum $[17,18]$.

Although in [14] no search for coexisting attractors was done (rather, the evolution of only the ECM with minimum carrier density was studied, for increased feedback level), the situation that two (or more) different cavity modes become unstable with increased feedback can also happen in short external cavities, and this behavior could be the explanation of the topological form of the chaotic attractor found in [6]. The competition between the state of minimum gain and the state of minimal linewidth has been reported in $[3,4]$, and the bistability of limit cycles supported by the same ECM has been demonstrated theoretically and experimentally in [13,9], but to our knowledge, this is the first report of multiattractor behavior in semiconductor lasers with optical feedback.

The Lyapunov exponents, calculated numerically using Farmer's technique [18], demonstrate that the dynamics of the coherent collapsed state is hyperchaotic, i.e., the laser has two or more positive exponents. Increasing the feedback rate, we find that the number of positive exponents becomes larger in a continuous way. From the spectrum of Lyapunov exponents, we calculated Kaplan and Yorke's dimension $[19,20]$ and metric entropy $[21]$ of the attractor. We find that the dimension increases abruptly at the critical point where the transition to the coherent collapsed state occurs, but for values of $k$ above this point, the dimension of the attractor increases roughly linearly. In addition, a continuous growth of the metric entropy indicates that the dynamics of the coherent collapsed state becomes more chaotic, as the feedback rate is augmented. These results which indicate an increase in degrees of freedom and complexity of the system are heuristically explained by the behavior found previously, i.e., that the number of cavity modes that are exited and the number of coexisting attractors increase linearly with the feedback rate.

This paper is organized as follows: in Sec. II a brief 
description of the theoretical model based on Lang and Kobayashi [1] delayed rate equations is given. In Sec. III the attractors originated after the ECM are analyzed using the Poincaré section and the Fourier spectrum. In Sec. IV the characterization of these attractors is done, by computing the Lyapunov spectrum, dimension, and entropy. Finally, Sec. V is devoted to a discussion of the results obtained.

\section{THE MODEL EQUATIONS}

The rate equations governing the behavior of a singlemode laser diode with weak to moderate feedback are the Lang and Kobayashi equations [1] for the amplitude $E_{0}(t)$ and the phase $\phi(t)$ of the electric field and the average carrier density $N(t)$ in the active region

$$
\begin{aligned}
& \frac{d E_{0}(t)}{d t}= \frac{1}{2}\left[G(N)-1 / \tau_{p}\right] E_{0}(t) \\
&+\frac{k}{\tau_{\text {in }}} E_{0}(t-\tau) \cos [\Delta(t)], \\
& \frac{d \phi(t)}{d t}= \frac{1}{2} \alpha\left[G(N)-1 / \tau_{p}\right]-\frac{k}{\tau_{\text {in }}} \frac{E_{0}(t-\tau)}{E_{0}(t)} \sin [\Delta(t)], \\
& \frac{d N(t)}{d t}=J-\frac{N(t)}{\tau_{s}}-G(N) E_{0}(t)^{2} .
\end{aligned}
$$

In these equations the field amplitude $E_{0}(t)$ is normalized such that $V_{c} E_{0}(t)^{2}$ is the total photon number in the laser waveguide (where $V_{c}$ is the volume of the active region). $\tau$ is the delay time ( $\tau=2 L / c$, where $L$ is the length of the optical path and $c$ is the velocity of light), $\tau_{s}$ is the carrier lifetime or population inversion lifetime, $\tau_{p}$ is the photon lifetime, and $\tau_{\text {in }}$ is the found-trip time in the laser cavity.

$$
\Delta(t)=\omega_{0} \tau+\phi(t)-\phi(t-\tau)
$$

is the phase delay (where $\omega_{0}$ is the laser frequency without feedback at the threshold of the laser operation). The linear gain per unit time is $G(N)=g\left(N-N_{0}\right)$, with $g$ being the modal gain coefficient and $N_{0}$ the carrier density at transparency. $k$ is the feedback parameter, i.e., $k^{2}$ is the power reflected from the external cavity relative to the power reflected from the laser mirror. $\alpha$ is the linewidth enhancement factor and $J$ is the current density in carriers per unit volume and unit time. Equations (1)-(3) do not include multiple reflection, and therefore are valid only for $k \ll 1$. The numerical values adopted for the parameters are $\alpha=4.4, g=1.1 \times 10^{-12} \mathrm{~m}^{3} / \mathrm{s}$, $N_{0}=1.1 \times 10^{24} \mathrm{~m}^{-3}, \tau_{s}=2 \mathrm{~ns}, \tau_{p}=2 \mathrm{ps}, \tau_{\text {in }}=6.7 \mathrm{ps}$, and $\tau=10 \mathrm{~ns}$. $J$ was taken as $J=2.0 J_{\text {th }}$, where $J_{\text {th }}=N_{\text {th }} / \tau_{s}$ and $G\left(N_{\text {th }}\right)=1 / \tau_{p}, J_{\text {th }}$ and $N_{\text {th }}$ being the threshold current density and carrier density $\left(J_{\text {th }}=7.77 \times 10^{32}\right.$ $\mathrm{m}^{-3} \mathrm{~s}^{-1}$ and $N_{\text {th }}=1.55 \times 10^{24} \mathrm{~m}^{-3}$ ). Finally, the feedback level $k$ is taken as a control parameter, which is varied to study the behavior of the laser.

The stationary solutions of Eqs. (1) - (3) are the external cavity modes of the laser. The stationary angular fre- quencies $\omega_{s}$ are the solutions of the equation

$$
\omega=\omega_{0}-\frac{k}{\tau_{\text {in }}} \sqrt{1+\alpha^{2}} \sin (\omega \tau+\arctan \alpha),
$$

while the stationary carrier density and field amplitude corresponding to a given value of $\omega_{s}$ are

$$
\begin{aligned}
& N_{s}=N_{\text {th }}-\frac{2 k}{g \tau_{\text {in }}} \cos \omega_{s} \tau, \\
& E_{s}^{2}=\frac{1}{g\left(N-N_{0}\right)}\left(J-\frac{N_{s}}{\tau_{s}}\right) .
\end{aligned}
$$

The stability of these solutions is determined by the value of the real part of the errors of the system determinant $D(s)[2]$. If

$$
1+\frac{k \tau}{\tau_{\text {in }}} \sqrt{1+\alpha^{2}} \cos (\omega \tau+\arctan \alpha)<0
$$
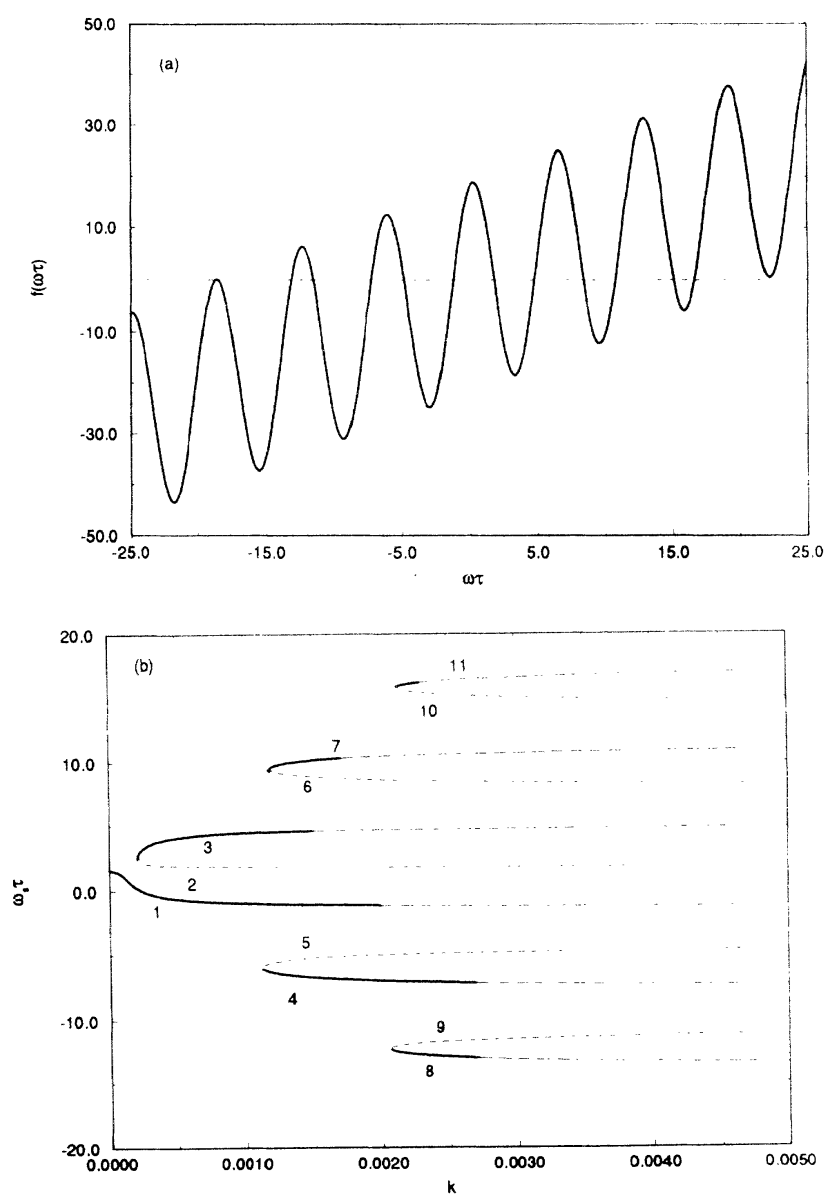

FIG. 1. (a) Graphical solution of Eq. (4) for $k=0.003$ and $\omega_{0} \tau=\pi / 2$.

$f(\omega \tau)=\omega \tau-\omega_{0} \tau-k \tau\left(1+\alpha^{2}\right)^{1 / 2} \sin (\omega \tau+\arctan (\alpha)] / \tau_{\text {in }} . \quad$ We observe that Eq. (4) has 11 roots for these parameters values. (b) Bifurcation diagram of the ECM as the feedback level is increased. The stationary angular frequencies $\omega_{s}$, i.e., the solutions of Eq. (4), are plotted as a function of $k$. The solid lines represent initially stable modes, the short-dashed lines represent saddle modes, and the long-dashed lines represent initially stable modes that become unstable after Hopf bifurcations. 
the determinant $D(s)$ has a zero with positive real part and the ECM is a saddle point. The angular frequencies of the ECM, i.e., the solutions of Eq. (4), are easily found by graphical construction [2]. Figure 1(a) shows the graphical construction for $k=0.003$, and we can observe that there are 11 ECM. Figure 1(b) shows the value of the stationary angular frequencies as a function of the feedback level $k$. We observe that, as $k$ increases, pairs of saddle-node bifurcations occur. In each saddle-node bifurcation a pair of EMC are created: one is an initially stable point (solid lien), while the other is a saddle point (short-dashed line). Modes labeled 1, 3, 4, 7, 8, and 11 are initially stable, while modes $2,5,6,9$, and 10 are unstable modes.

In addition to condition (7), a pair of complexconjugate zeros of a stable mode can move to the righthalf $s$ plane. In this case the stable mode become unstable in a Hopf bifurcation, and a limit cycle appears. In Fig. 1(b) these modes are represented by long-dashed lines after the Hopf bifurcation.

\section{DYNAMICS OF THE MODEL}

The evolution of each initially stable ECM shown in Fig. 1(b) is studied, for an increasing feedback level. Equations (1)-(3) were integrated using a standard sixthorder Runge-Kutta integration routine with a time increment $\Delta t=10 \mathrm{ps}$. The initial conditions are taken from Eqs. (4)-(6), and the value of the electric field and phase are stored in memory in order to use them in the next round-trip interval. We analyzed the trajectory (after a certain number of round rips in order to eliminate transient effects) using the Poincaré section technique $[15,16]$. The Poincaré sections were obtained plotting the normalized carrier density $\left[N(t) / N_{\text {th }}-1\right]$ and the phase delay $[\phi(t)-\phi(t-\tau)]$ at the intersection points of the trajectory with the plane

$$
I(t) / I_{\mathrm{sol}}=E(t)^{2} / E_{\mathrm{sol}}^{2}=1 \text {. }
$$

As the feedback level is increased, the initially stable modes become unstable and give way to limit-cycle behavior after a Hopf bifurcation. For an increased feedback rate, the interval of stable-limit cycle dynamics is followed by a two-frequency quasiperiodic regime after a secondary Hopf bifurcation. The transition scenario to chaos of the two-frequency regime is different for each external cavity mode. Table I summarizes the values of the feedback rate at which the bifurcations occur, for each ECM studied.
In Fig. 2 we illustrate the evolution of several coexisting attractors, as the feedback level is increased (the symbol $O$ indicates the projection on the Poincaré plane of the unstable ECM that originated attractor). For $k=0.03$, attractor A (originated from the ECM labeled 1 in Fig. 1), B (originated from mode 3), C (originated from mode 4), and F (originated from mode 11) are two-tori, attractor D (originated from mode 7 ) is a limit cycle, and mode 8 is still a stable fixed point. For $k=0.0035$, attractors A, B, C, D, and E (originated from mode 8) are all two-tori, while attractor $F$ became unstable. The Poincaré section of attractor $\mathrm{D}$ becomes complicated for $k=0.004$, and it looks chaotic for $k=0.0045$. For $k=0.005$, attractors A, C, D, and E are unstable and the only stable attractor (besides the ECM's) is attractor B. As an example, Fig. 3 shows the evolution of an initial condition close to attractor $E$. The arrows indicate the direction of evolution: the trajectory spends a while in the vicinity of attractors E, C, and A until it finally arrives in the vicinity of stable attractor $B$, where it remains.

We will now study in detail the characteristics and evolution of attractors A and B, which were originated from the ECM 1 and 3, respectively. Figure 4 illustrates the evolution of attractor A. [In Figs. 4 and 5 a different Poincaré plane is used. We plot the intersection points of the trajectory with the plane $N(t) / N_{\text {th }}-1=0$.] Torus A undergoes a second Hopf-Landau bifurcation and a three-dimensional torus appears. This three-frequency regime is stable until two frequencies lock and attractor $A$ is again a two-dimensional torus. If we continue to increase the amount of feedback, the attractor loses stability and the system switches to the coexisting attractor B.

The evolution of attractor $B$ is illustrated in Fig. 5 . Torus $B$ undergoes two period-doubling bifurcations and its Poincaré section becomes very complicated before becoming chaotic. As we can observe in Figs. 2, 4, and 5, attractors $A$ and $B$ are topologically very different. While the Poincaré section of torus $\mathbf{A}$ has phase delay $\phi(t)-\phi(t-\tau)<0$, the Poincaré section of torus B has phase delay $\phi(t)-\phi(t-\tau)>0$. The Fourier spectrum (Fig. 6) shows that tori A and B have the same incommensurate frequencies $f_{1}$ and $f_{2} . f_{1}$ is the relaxation frequency of the laser, while $f_{2}$ is of the order of $1 / \tau$ in agreement with the analysis of [12]. Therefore, attractors A and B have similar Fourier spectrum but they are easily distinguished by calculating their Poincaré section.

Figure 7 shows the evolution of attractor $B$ for higher

TABLE I. Critical values of the feedback rate at which bifurcations occur, for each external cavity mode studied.

\begin{tabular}{ccccc}
\hline \hline Attractor & ECM & $\begin{array}{c}\text { First Hopf } \\
\text { bifurcation }\end{array}$ & $\begin{array}{c}\text { Second Hopf } \\
\text { bifurcation }\end{array}$ & $\begin{array}{c}\text { Attractor loses } \\
\text { stability }\end{array}$ \\
\hline A & 1 & 0.0021 & 0.0022 & 0.0049 \\
B & 3 & 0.0017 & 0.0029 & 0.0055 \\
C & 4 & 0.0028 & 0.0029 & 0.0046 \\
D & 7 & 0.0018 & 0.0031 & 0.0046 \\
E & 8 & 0.0033 & 0.0034 & 0.0046 \\
F & 11 & 0.0023 & 0.0025 & 0.0030 \\
\hline \hline
\end{tabular}



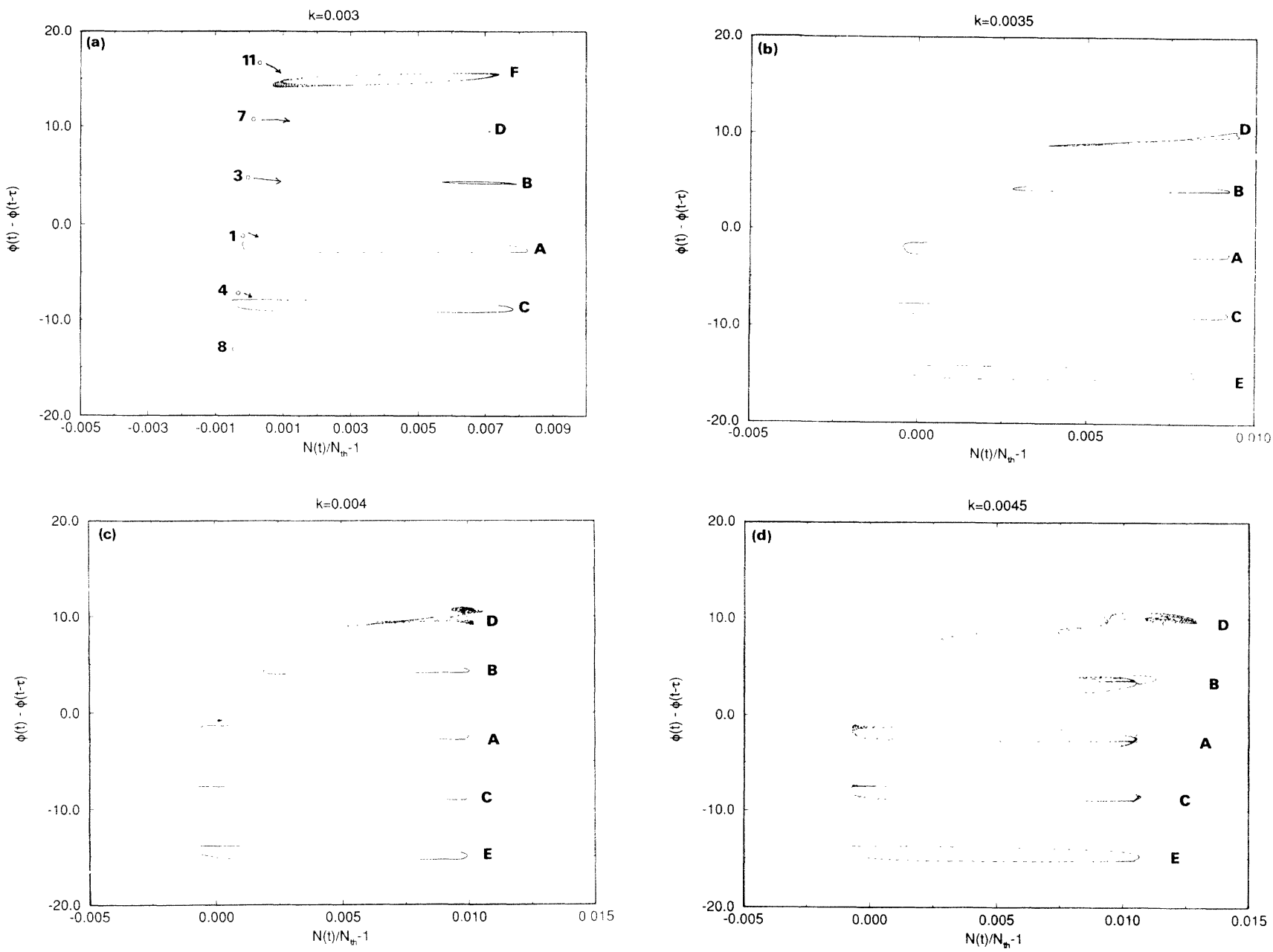

FIG. 2. (a) Coexistence of attractors for $k=0.003$. The symbol $\bigcirc$ indicates the projections of the external cavity modes on the Poincare plane, and the arrows indicate the final state of the laser when one of the ECM is taken as an initial condition. To construct the Poincare sections, we plot the intersection points of the trajectories with the plane $[E(t)]^{2} / E_{\text {sol }}^{2}=1$. (b) Coexistence of attractors for $k=0.0035$. (c) Coexistence of attractors for $k=0.004$. (d) Coexistence of attractors for $k=0.0045$.

values of the feedback. For $k=0.0054$, the attractor is a complicated two-dimensional torus that gradually becomes chaotic [see, for example, the Poincaré section shown in Fig. 7(b)]. For an increased feedback rate the attractor loses stability and a switching between the coexisting unstable attractors $A$ and $B$ occurs. The phenomenon is strongly reminiscent of the intermittence phenomenon, since the trajectory jumps randomly from the vicinity of the unstable torus $A$ to the vicinity of tours B. Notice that attractor B is still more stable than attractor A, since the Poincaré section shown in Fig. 7(c) has more points close to attractor B than to attractor $\mathrm{A}$. If we continue to increase the feedback, in the "fully developed" coherent collapsed state, the trajectory jumps between all attractors that were originated as the initially stable ECM becomes unstable.

Although the coexistence of attractors encountered here is in the case of a large external cavity, it can also happen in a smaller cavity, and this behavior might be the explanation of Fig. 1(e) of [14], where the Poincare section of the chaotic attractor shown clearly has two sectors, one with points with positive phase delay and the

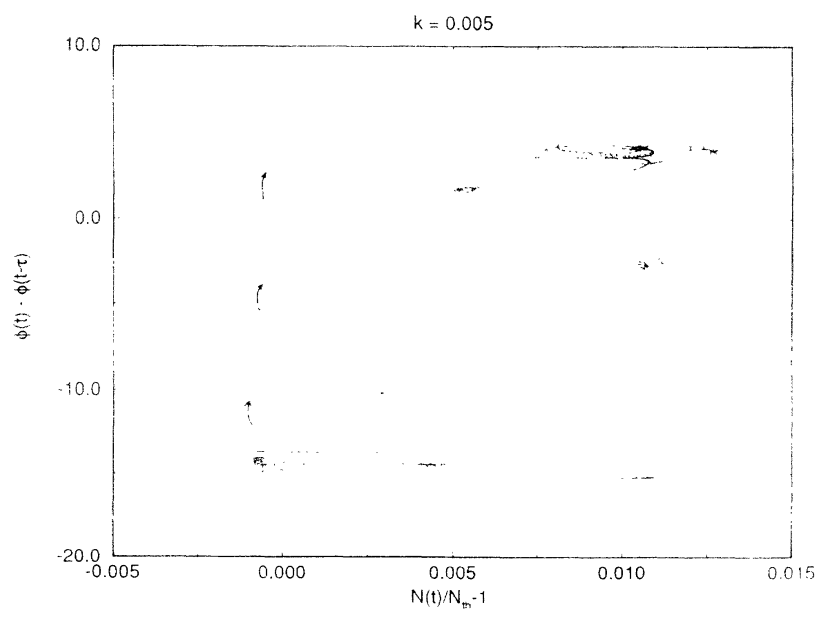

FIG. 3. Evolution of an initial condition in the vicinity of unstable attractor $\mathrm{E}$, for $k=0.005$. The trajectory evolves to a region close to the unstable attractor $\mathrm{C}$, then to attractor $\mathrm{A}$, and finally arrives at the neighborhood of attractor $\mathrm{B}$, which is a stable attractor for this value of $k$. 

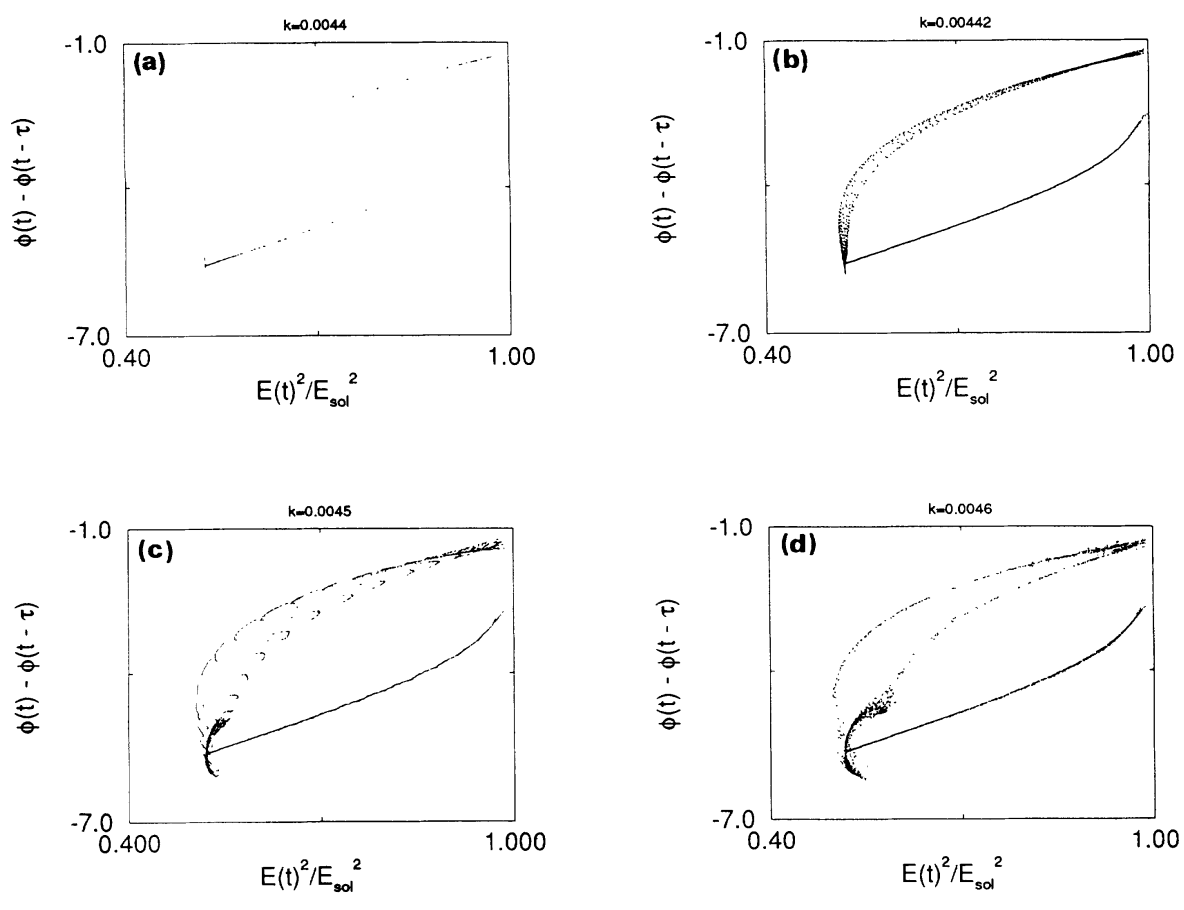

FIG. 4. Poincaré section of attractor $\mathrm{A}$ for (a) $k=0.0044$, (b) 0.00442, (c) 0.0045, (d) 0.0046 , (e) 0.00465 , and (f) 0.0049 . The Poincaré sections were obtained plotting the normalized intensity $\left[(E(t)]^{2} / E_{\text {sol }}^{2}\right)$ and the phase delay $[\phi(t)-\phi(t-\tau)]$ at the intersection points of the trajectory with the plane $N(t) / N_{\text {th }}-1=0$.
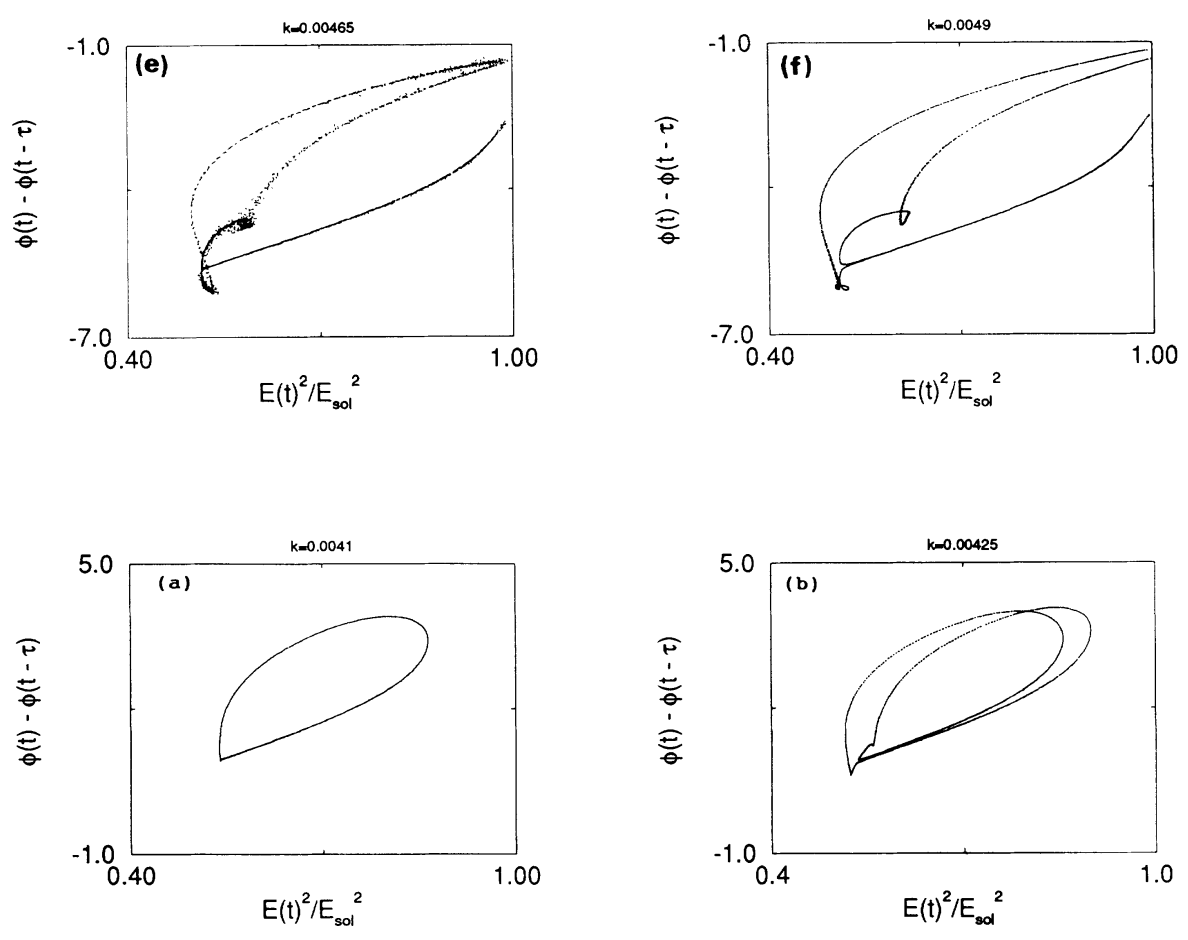

FIG. 5. Poincaré section of attractor $B$ (see the caption of Fig. 4: (a) $k=0.0041$, (b) 0.00425 ; (c) 0.0044 , and (d) 0.0049 .
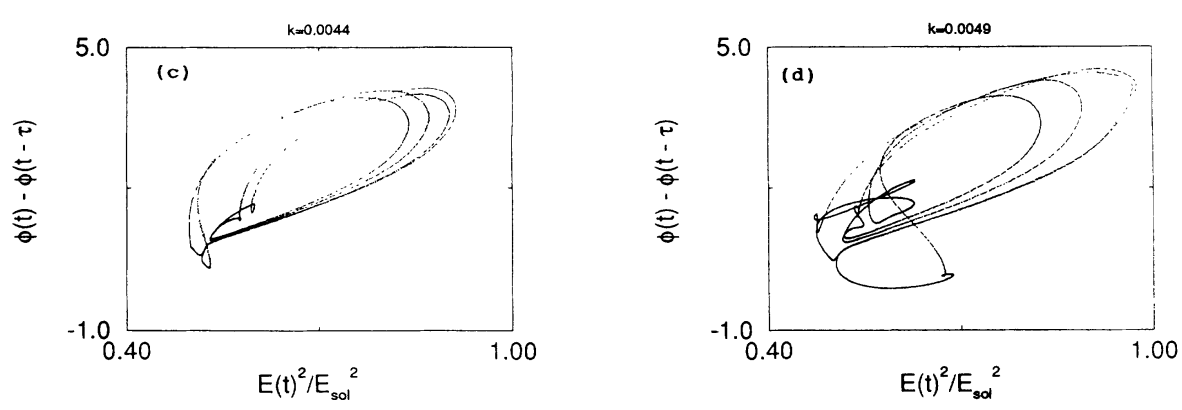
other with points with negative phase delay.

Let us now comment briefly on the dynamics of the laser when the external cavity length is varied for a fixed feedback rate. For $k=0.006$ and low values of the delay times $\tau$, a rich variety of bifurcations is found, with the creation and annihilation of several low-dimensional attractors (limit cycles, two-torus). As $\tau$ is increased, for most values of $\tau$ the attractors generally become more complicated, i.e, they are three-torus, or period-doubled torus. For even higher values of $\tau$, the attractors become unstable and the chaotic state studied previously is the only stable attractor found. In Fig. 8 some typical Poincaré sections are shown, for $k=0.006$ and an increasing value of $\tau$. For $\tau=1$ and $3.3 \mathrm{~ns}$, the attractor shown is a two-torus; for $\tau=4 \mathrm{~ns}$, the attractor is a torus that period doubled; for $\tau=5 \mathrm{~ns}$, the attractor is a three-torus; and for $\tau=7$ and $9 \mathrm{~ns}$, the attractor is chaotic. Notice that the complexity of the attractors increase when the delay
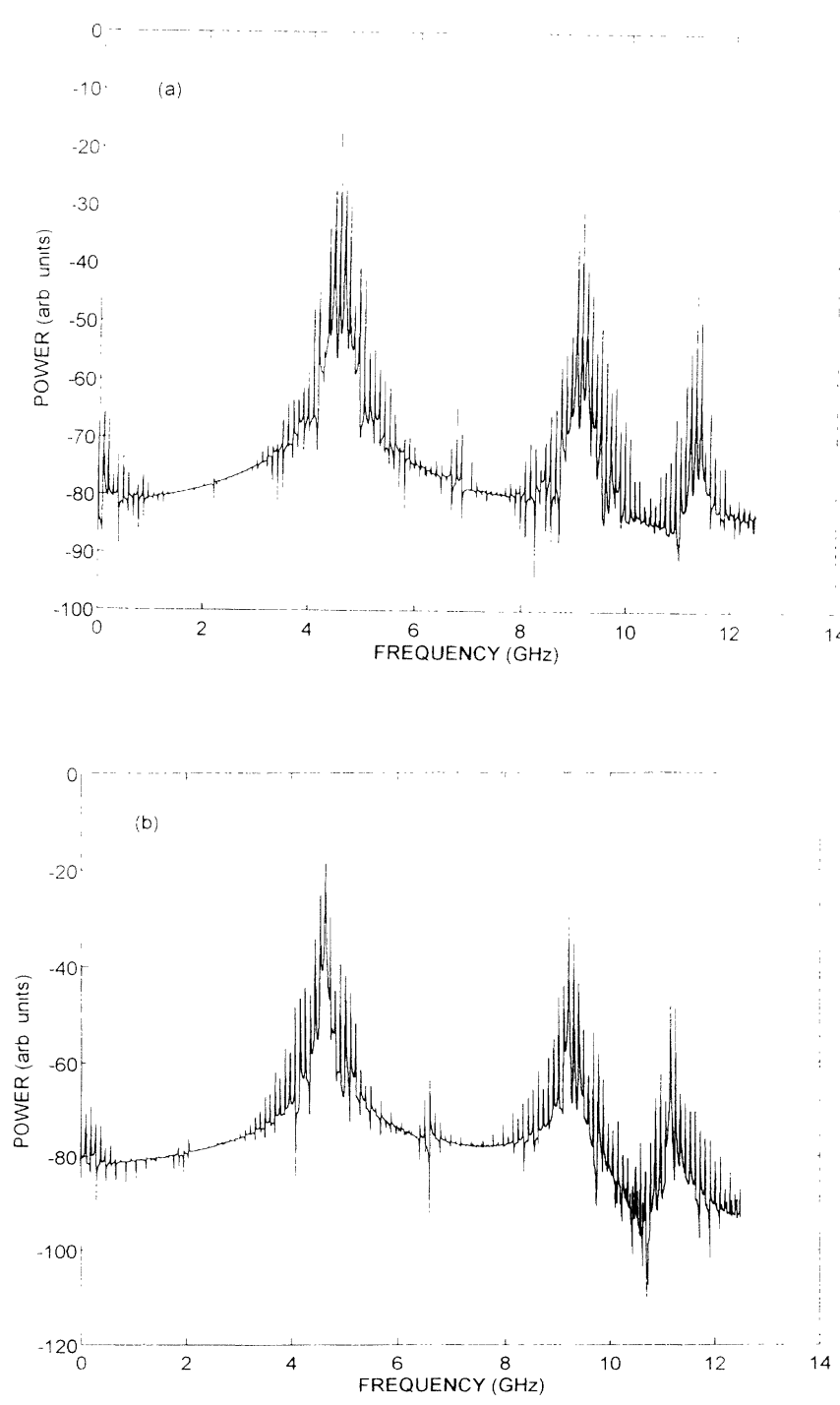

FIG. 6. Fourier spectrum of attractors A and B calculated from the time evolution of the time evolution of the normalized light intensity $\left[(E(t)]^{2} / E_{\text {sol }}^{2}\right)$. time is augmented. The study of the origin and evolution of the attractors of the system when the delay time is varied for a fixed feedback rate will be the object of future work.

Since the results about Lyapunov exponents and dimensionality of the coherent collapsed state that will be presented in Sec. IV rely on the numerical integration of Eqs. (1)-(3), an important point to discuss here is the numerical accuracy and convergence of the integration procedure. A typical power spectrum (see, for example, Fig. 6) indicates that Eqs. (1)-(3) are a still set of equations. Stiffness occurs when the dynamics has two or more very different characteristic time scales. In our case the two characteristic time scales are the fast oscillating component $\tau_{\text {relax }} \approx 0.20 \mathrm{~ns}$, which is related to the frequency of the relaxation oscillations of the solitary laser $\left(f_{1} \approx 5\right.$ $\mathrm{GHz}$, and the slow oscillating component $\tau_{\text {ext }} \approx 10 \mathrm{~ns}$, which is related to the round-trip frequency determined by the length of the external cavity $\left(f_{2} \approx c / 2 L \approx 0.1\right.$ $\mathrm{GHz}$ ).

After a few tests it was found that an interval $\Delta t=10$ ps, although fairly large as compared with the characteristic time scale of the optical field $(2 \mathrm{ps})$, was still acceptable to obtain sufficiently accurate convergence to the solutions (this value is to be compared with the fast oscillating component, i.e., the period of the relaxation oscillation, $\approx 200 \mathrm{ps}$ ). Finally, an interval of $1000 \mathrm{~ns}$ (corresponding to 100 round trips in the external cavity) was found to be sufficient in order to eliminate transient effects (this value is to be compared with the slow oscillating component, i.e., the delay time $\tau=10 \mathrm{~ns}$ ).

\section{LYAPUNOV EXPONENTS, DIMENSION, AND ENTROPY}

In this section we turn to study the characterization of attractors $\mathrm{A}, \mathrm{B}$, and the coherent collapsed state. The Lyapunov exponents for an attractor show qualitatively the sensitive dependence on initial conditions by measuring the average separation speed of nearby trajectories on the attractor. The fractal dimension of an attractor is related to the number of collective degrees of freedom exited in the system, and the metric entropy, which is the sum of the positive Lyapunov exponents, provides information on a typical predictability time of the motion. Regular motions are associated with an integer value of the dimension, and with zero entropy, chaotic systems have fractal dimension and positive finite entropy and stochastic processes have infinite dimension and entropy.

Since the laser diode with feedback from an external cavity is a system with infinite dimensions, to calculate the Lyapunov exponents, we must study the evolution of infinitesimal perturbations that are vectors with three components, two of which (the field and the amplitude) are functions of time over the entire delay $\tau$. The technique we used is as follows $[14,18]$ : for each exponent $\lambda^{i}$ to be computed, we arbitrarily selected an initial perturbation

$$
d \mathbf{x}^{i}(0)=\left(d E^{i}(0), d \phi^{i}(0), d N^{i}(0)\right) .
$$

We monitored the evolution of the trajectory 

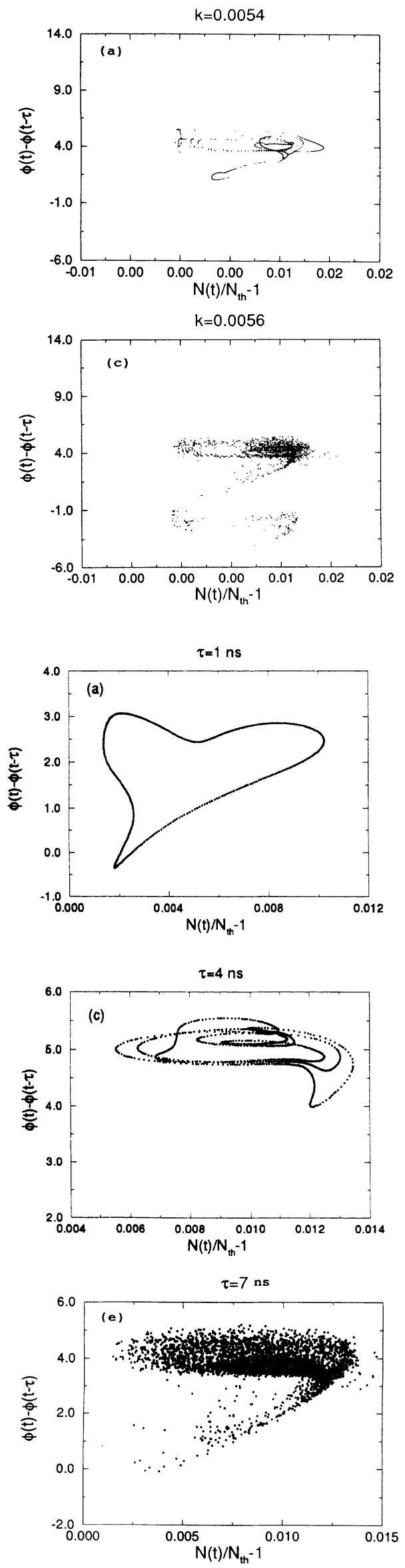
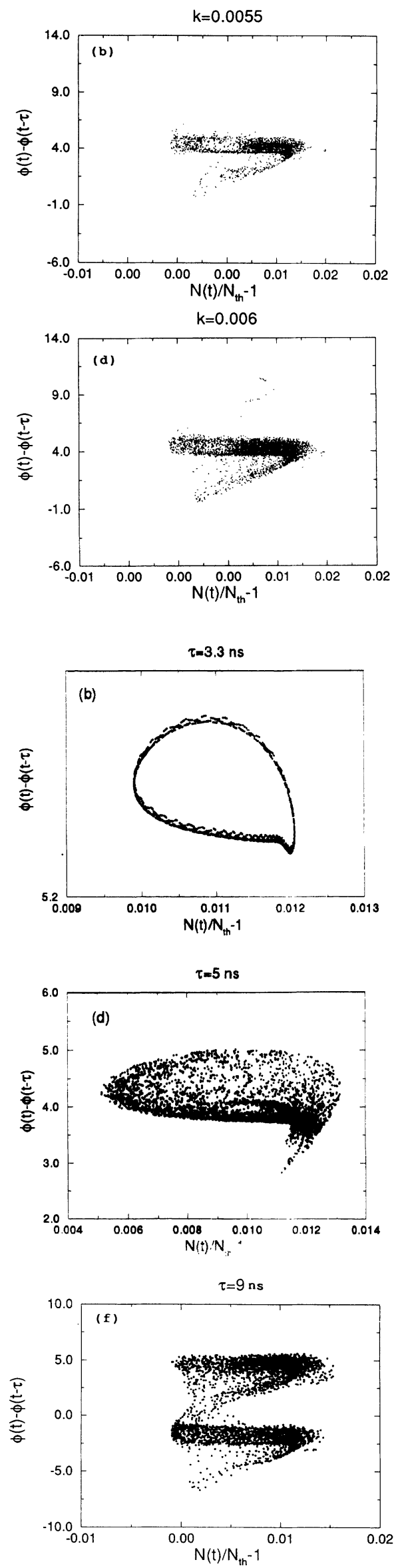

FIG. 7. Poincaré section of attractor B (The Poincaré plane used is $\left.[E(t)]^{2} / E_{\mathrm{sol}}^{2}=1\right)$ : (a) $k=0.0054$, (b) 0.0055 , (c) 0.0056 , and (d) 0.006 .
FIG. 8. Poincaré sections for $k=0.006$ and (a) $\tau=1 \mathrm{ns,} \mathrm{(b)}$ $\tau=3.3 \mathrm{~ns}$, (c) $\tau=4 \mathrm{ns,} \mathrm{(d)} \tau=5$ ns, (e) $\tau=7 \mathrm{ns,} \mathrm{and} \mathrm{(f)} \tau=9 \mathrm{~ns}$. 


$$
\mathbf{x}(t)=(E(t), \phi(t), N(t))
$$

and of the perturbations $\mathbf{d x} \mathbf{x}^{i}(t)$, integrating simultaneously the nonlinear evolution equations (1)-(3) as well as the linearized equations, for a round-trip time $\tau$. Then we normalized the first perturbation $\mathbf{d x}^{1}$ to have length 1 and using a Gram-Schmidt algorithm, we orthonormalized the second vector relative to the first, the third relative to the first and second, and so on. We repeated this procedure for $L$ round-trip times and computed

$$
\lambda^{i}=\frac{1}{L \tau} \sum_{k=1}^{L} \ln \left\|d x^{i}(k)\right\|,
$$

where the Euclidean metric was chosen to define distance in the phase space

$$
\begin{aligned}
\left\|d x^{i}(k)\right\|^{2}= & \frac{1}{n} \sum_{j=1}^{n}\left[\frac{d E_{j}^{i}(k)}{E_{0}}\right]^{2} \\
& +\frac{1}{n} \sum_{j=1}^{n}\left[d \phi_{j}^{i}(k)\right]^{2}+\left(\frac{d N^{i}(k)}{N_{0}}\right)^{2} .
\end{aligned}
$$

Here, $d E_{j}^{i}$ and $d \phi_{j}^{i}$ with $j=1, \ldots, n$ are the electric-field and phase components of the $i$ perturbation over the entire delay interval $\tau$.

Since Eqs. (1) - (3) are invariant under a global translation in the phase $\left(\phi_{j} \rightarrow \phi_{j}+a\right)$, two points in the attractor that differ in a constant value of $\phi$ will not merge nor separate. Thus, the system has a "spurious" zero Lyapunov exponent in the spectrum that corresponds to a fixed zero in the system determinant $D(s)$ [2]. This exponent does not influence the stability and will be ignored in the rest of our calculations.

In Fig. 9 the nine largest Lyapunov exponents of attractor $\mathrm{A}$ are shown, as a function of the amount of feedback $k$. For $k \leq 0.0044$, the attractor is a two-torus with two exponents $\lambda^{1}, \lambda^{2}=0$, while for $k>0.0044$, the attractor is a three-torus that has three exponents $\lambda^{1}, \lambda^{2}, \lambda^{3}=0$. At the transition from the three-torus to the two-torus, when frequency locking occurs, $\lambda^{1}$ becomes slightly positive. After frequency locking, this exponent becomes negative and the attractor is a two-torus again. Finally,

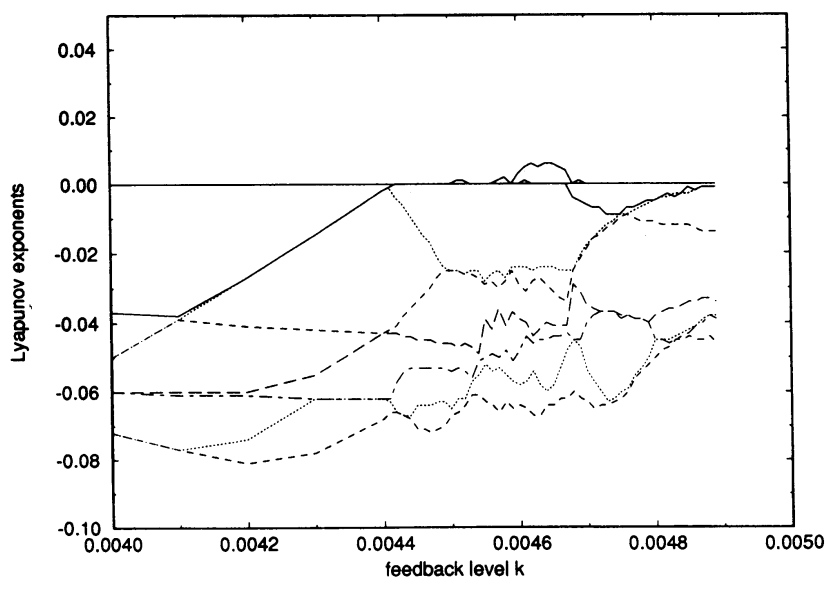

FIG. 9. Lyapunov exponents of attractor $\mathrm{A}$ as a function of $k$ at $k=0.0049$, attractor $\mathrm{A}$ becomes unstable as two Lyapunov exponents approach zero.

Figure 10 shows the nine largest exponents of torus $B$. For $k<0.0055$, torus B has two exponents $\lambda^{1}, \lambda^{2}=0$. We observe two period-doubling bifurcations that occur when the third exponent becomes zero. At the critical point when the transition to the chaotic state occurs, $\lambda^{1}$ and $\lambda^{2}$ abruptly become positive, indicating that the laser presents hyperchaotic behavior. In the "fully developed" coherent collapsed state (for large values of $k$ ), the number of positive Lyapunov exponents (see Fig. 12) grows approximately linearly with the feedback rate.

In addition, from the spectrum of Lyapunov exponents we calculated the Lyapunov dimension using the definition of Kaplan and Yorke [19]

$$
D_{L}=j+\frac{\sum_{i=1}^{j} \lambda^{i}}{\left|\lambda^{j+1}\right|},
$$

where $\lambda^{1}>\lambda^{2}>\cdots>\lambda^{n}$ and $j$ is the largest integer for which $\lambda^{1}+\lambda^{2}+\cdots+\lambda^{j} \geq 0$. For typical attractors it has been conjectured [20] that the Lyapunov dimension is equal to the well-known information dimension.

The Lyapunov dimension of attractor $A$ is plotted in Fig. 11 for $k$ between 0.004 and 0.0049 (the dots indicate the number of non-negative exponents of the attractor). For $k<0.0044$, the attractor has two exponents $\lambda^{1}, \lambda^{2}=0$, and the Lyapunov dimension is equal to 2 . At $k=0.0044$, a Hopf-Landau bifurcation occurs and a three-torus appears. The attractor has then $\lambda^{1}, \lambda^{2}, \lambda^{3}=0$ and the dimension is equal to 3 . Note that before frequency locking occurs, the dimension exceeds 3 , while there are only three non-negative exponents. For $k \geq 0.0047$, the attractor is again a two-dimensional torus with two zero Lyapunov exponents and the dimension decreases to 2 again.

Figure 12 shows the Lyapunov dimension of attractor $\mathrm{B}$, as a function of the feedback $k$ (the dots indicate the number of non-negative exponents of the attractor). We observe that the dimension of the attractor is equal to 2

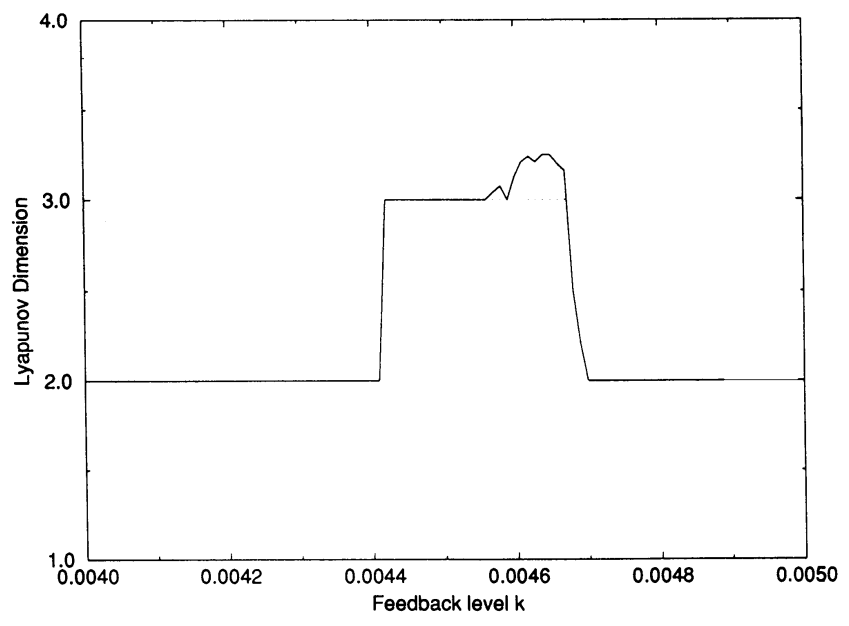

FIG. 10. Lyapunov exponents of attractor B as a function of 


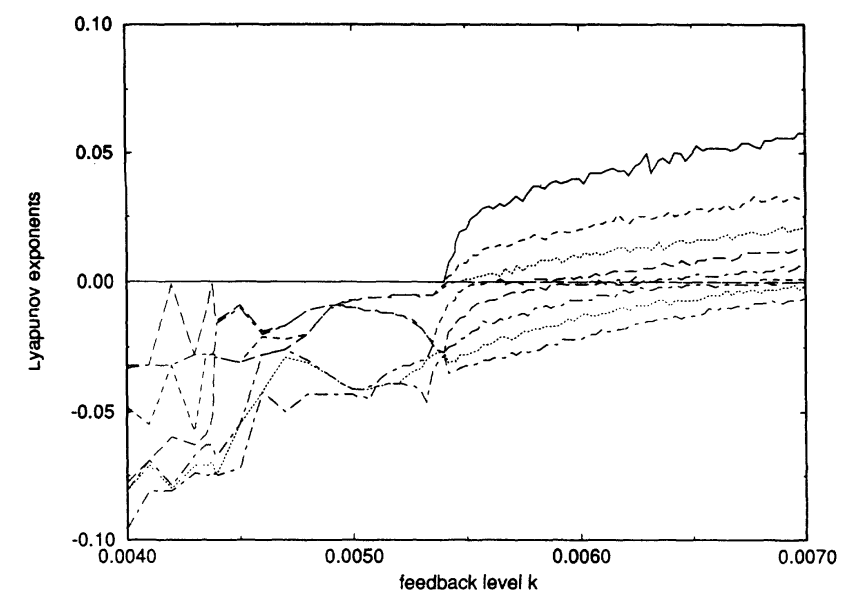

FIG. 11. Dimension of attractor A, calculated from Eq. (10). The Lyapunov dimension is shown in a solid line, while the number of non-negative Lyapunov exponents is indicated by a dotted line.

until the transition to the coherent collapsed state, where the dimension increases abruptly, indicating fractal behavior. If we continue to increase the feedback level above the critical point, the dimension increases roughly linearly, as a consequence of the fact that the Lyapunov exponents of the attractor become positive in a continuous way.

Since chaotic trajectories continually generate new information, the metric entropy provides a quantitative way to measure this information and to describe "how chaotic" a system is. The relation between the metric entropy and the Lyapunov exponents [21] of an attractor is

$$
h_{\mu}=\sum_{i=1}^{j} \lambda_{+}^{i},
$$

where $h_{\mu}$ is the metric entropy and $\lambda_{+}^{i}$ are the positive Lyapunov exponents of the attractor. Figure 13 shows the metric entropy of attractor $\mathrm{B}$ as a function of $k$. We

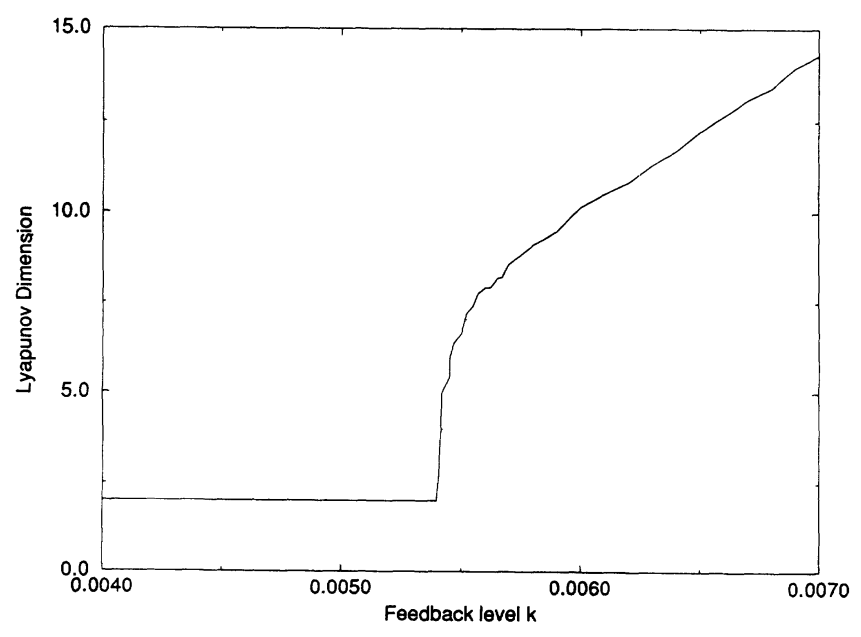

FIG. 12. Dimension of attractor B, calculated from Eq. (10). The solid line is the Lyapunov dimension, while the dots indicate the number of non-negative Lyapunov exponents.

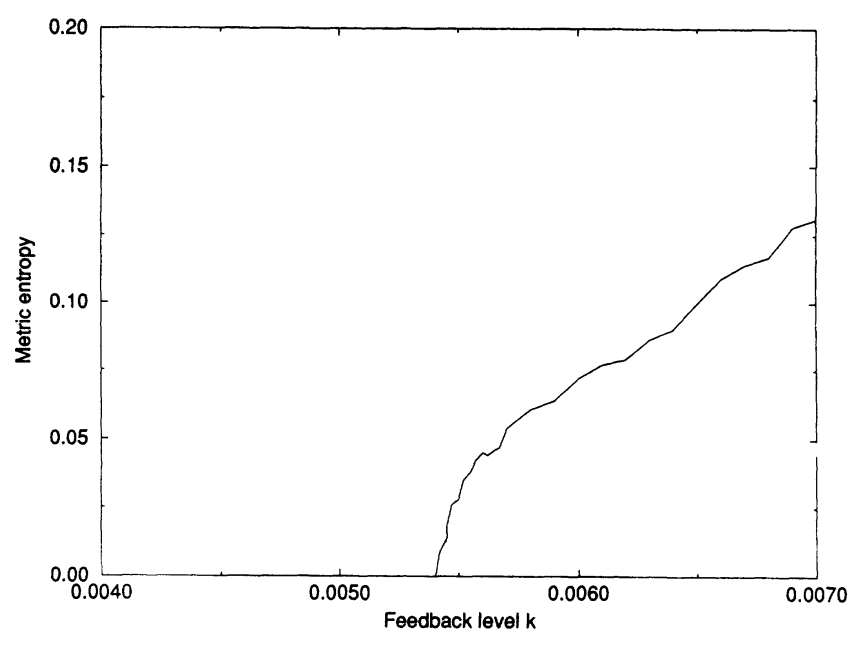

FIG. 13. Metric entropy of attractor B, calculated according to Eq. (11).

observe that, for values of the feedback below the critical point, the metric entropy is zero, indicating regular behavior. At the transient to the coherent collapsed state, the entropy increases abruptly, but for values of $k$ exceeding the critical value, the entropy increases almost linearly with the feedback rate, indicating a continuous grow of the dynamical complexity of the system with the feedback rate.

The hyperchaotic behavior and the high dimensionality found are explained qualitatively in terms of the result found in Sec. III. As the feedback rate increases, the number of ECM that become unstable grows and, as a consequence, the number of degrees of freedom and dynamical complexity of the system increases. In addition, this behavior might also be related to the fact that the external cavity is very large, since it is well known that the dimension of the attractors of differential delay systems increases with delay time (see, for example, Fig. 8 , where the dimension of the attractors generally increases, as $\tau$ is augmented). In [14], where a smaller external cavity was used, the fractal dimension found is slightly smaller than 4 and no hyperchaotic behavior was encountered, i.e., only one Lyapunov exponent became positive at the transition to coherent collapsed state. An in-depth investigation of the high dimensionality of the laser, shown in Figs. 10 and 11, and the relation with the external cavity length is in progress and will be reported elsewhere.

\section{SUMMARY AND CONCLUSIONS}

In summary, the laser dynamics induced by delay feedback from a distant reflector has been analyzed in detail. The coexistence of several attractors is found. We show that these attractors are originated after Hopf bifurcations of different initially stable ECM of the laser. When the transition to the coherent collapsed state occurs, the trajectory jumps between these unstable attractors and the phenomenon of intermittence appears.

The calculation of the spectrum of Lyapunov ex- 
ponents demonstrates the hyperchaotic nature of the coherent collapsed state. The fact that a large number of exponents become positive for increasing values of $k$ is in agreement with the results found previously, i.e., that a large number of EMC of the laser became unstable as $k$ is varied, giving rise to periodic and quasiperiodic behavior before the onset of chaotic dynamics. From the spectrum of Lyapunov exponents we calculated an estimation of the fractal dimension and entropy of the coherent collapsed state. We find that, at the critical point when the transition occurs, the dimension and entropy of the attractor increase abruptly, while they increase almost linearly above this point. These results indicate a continuous increase of the degrees of freedom and the dynamical complexity of the state, when the feedback rate is augmented. A qualitative explanation of the behavior found is that the number of degrees of freedom is proportional to the number of external cavity modes which might be exited and thus increases linearly with the feedback level.

\section{ACKNOWLEDGMENTS}

The author would like to thank Dr. A. Sicardi and M.Sc. C. Cabeza for useful discussions and suggestions. This work was supported in part by PEDECIBA (Project No. URU/84/002-UNDP).
[1] R. Lang and K. Kobayashi, IEEE J. Quantum Electron. QE-16, 347 (1980).

[2] B. Tromborg, J. H. Osmundsen, and H. Olesen, IEEE J. Quantum Electron. QE-20, 1023 (1984).

[3] J. Mork, B. Tromborg, and P. L. Christiansen, IEEE J. Quantum Electron. QE-24, 123 (1988).

[4] N. Schunk and K. Petermann, IEEE J. Quantum Electron. QE-24, 1242 (1988).

[5] G. C. Dente, P. S. Drukin, K. A. Wilson, and C. E. Moeller, IEEE J. Quantum Electron. QE-24, 2441 (1988).

[6] D. Baums. W. Elsässer, and E. O. Göbel, Phys. Rev. Lett. 63, 155 (1989).

[7] J. Sacher, W. Elsässer, and E. O. Göbel, Phys. Rev. Lett. 63, 2224 (1989).

[8] J. Mork, J. Mark, and B. Tromborg, Phys. Rev. Lett. 65, 1999 (1990).

[9] J. Mork, B. Tromborg, and J. Mark, IEEE J. Quantum Electron QE-28, 93 (1992).

[10] J. Sacher, D. Baums, P. Panknin, W. Elsässer, and E. O. Göbel, Phys. Rev. A 45, 1893 (1992).

[11] Jun Ye, Hau Li, and John G. McInerney, Phys. Rev. A 47,
2249 (1993).

[12] A. Ritter and H. Haug, J. Opt. Soc. Am. B 10, 130 (1993); 10, 145 (1993).

[13] A. Ritter and H. Haug, IEEE J. Quantum Electron. QE-29, 1064 (1992).

[14] C. Masoller, A. C. Sicardi Schiffino, and C. Cabeza, Opt. Commun. 100, 331 (1993).

[15] P. Bergé, Y. Pomeau, and C. Vidal, Order within Chaos (Wiley, New York, 1984).

[16] H. G. Schuster, Deterministic Chaos, 2nd. ed. (VCH, Weinheim, Germany, 1988).

[17] G. Benettin, L. Galgani, and J.-M. Strelcyn, Phys. Rev. A 14, 2338 (1976).

[18] J. D. Farmer, Physica D 4, 366 (1982).

[19] J. Kaplan and J. Yorke, Functional Differential Equations and Approximation of Fixed Points, edited by H. O. Peitgen and H. O. Walther (Springer, Berlin, 1979), p. 228.

[20] J. D. Farmer, E. Ott, and J. A. Yorke, Physica D 7, 153 (1983).

[21] J. P. Eckmann and D. Ruelle, Rev. Mod. Phys. 57, 617 (1895). 